\title{
Venous thromboembolism prophylaxis usage in critical care units in National Hospital Sri Lanka
}

\author{
*I. A. C. L. Perera \\ Registrar, Anaesthesiology, National Hospital of Sri Lanka \\ *Corresponding author : perera.lakmini@yahoo.com
}

Key words: venous thromboembolism, critically ill

\begin{abstract}
Background:
Venous thromboembolism (VTE) is an important cause of mortality and morbidity in critical care patients. This is as a result of high prevalence of VTE risk factors in this group of patients including physical inactivity, immobilization, vascular injury and / or hypercoagulable state. The effect of these risk factors are cumulative ${ }^{1,2,3}$. VTE may progress into thrombophebitis or pulmonary embolism (PE) with increased mortality and morbidity and have enormous economic issues. But the risk of VTE and its prevention have been poorly characterized in this population. Evidence based thromboembolic prophylaxis guidelines are also not widely available for these critically ill patients ${ }^{3,4}$.

The main objectives of this audit were to determine the presence and the type of the risk factors and the prophylaxis given in patients admitted to five adult intensive care units (ICU) in National Hospital Sri Lanka (NHSL).
\end{abstract}

Method:

This audit was carried out from $1^{\text {st }}$ to $31^{\text {st }}$ of March 2011 in five critical care units in NHSL. Confidentiality was maintained. Data was obtained with the use of pre formed data collection forms, filled by post graduate trainees in anaesthesiology.

\section{Results:}

There were a total of 90 patients, $53 \%$ male and $47 \%$ female, and $40 \%$ were in the $30-40$ year age group. Majority (49\%) of the patients were admitted to ICU following surgery. 35 patients (38.9\%) had at least 2 risk factors for VTE. 39 patients (43.3\%) had received VTE prophylaxis. 51 patients $(56.6 \%)$ did not get any prophylaxis against VTE. $43.3 \%$ were given only pharmacological prophylaxis and $33.3 \%$ were offered both pharmacological and mechanical prophylaxis. Enoxaparin 40mg daily was used as the drug of choice in majority of the patients irrespective of their body weight. Pharmacological VTE prophylaxis for the postoperative patients was started 8-12 hours after admission in the majority.

\section{Conclusion:}

We found an under usage of VTE prophylaxis on at risk patients in intensive care units at NHSL. We would like to highlight the importance of having institutional guidelines on VTE prophylaxis and improving the awareness on VTE among the ICU staff.

\section{Background:}

VTE is a major cause of mortality and morbidity in both hospital and community setting, causing an estimated 60,000 deaths per year in $\mathrm{UK}^{5}$. Within this population up to 25,000 are potentially preventable with implementation of VTE preventive strategies. ${ }^{5}$. Deep vein thrombosis (DVT) and PE contribute significantly to mortality and morbidity associated with critical illness.
Among patients who died in ICU, PE has been reported in $7-27 \%$ (mean 13\%) of post mortem examinations, and PE was thought to have

caused or contributed to death in $0-12 \%$ (mean $3 \%$ ); although a clinical suspicion on $\mathrm{PE}$ was present in only $30 \%$ of these patients before death $^{3}$. Cross- sectional studies of medical and 
surgical intensive care unit patients have shown that approximately $10 \%$ of the patients have DVT on admission to the $\mathrm{ICU}^{4}$. The majority of patients admitted to the critical care units, carries major risk factors for VTE and most have multiple risk factors. Many of these risk factors for VTE precede ICU admission while in others they develop during the course of their stay in ICU. Advancing age, recent surgical procedures, malignancy, trauma and past history of DVT are some of the risk factors they carry on admission. Patients are further predisposed to DVT during their ICU stay due to prolonged immobilization, sepsis, vascular injuries from indwelling central venous catheters and other invasive interventions, heart failure, mechanical ventilation and paralysis. The importance of each of these clinical risk factors is unknown, as is the role of inherited or acquired coagulation system abnormalities ${ }^{3,4}$.

Pharmacological and/or mechanical prophylaxis against DVT is recommended for this high risk population. These have shown efficacious in preventing DVT compared with no prophylaxis. 14 studies over this matter have reported that compliance with some form of thromboprophylaxis occurred in 33-100\% of critically ill patients in ICU ${ }^{3}$.

The main objectives of this audit were to determine the presence and the type of the risk factors and the prophylaxis given in patients admitted to five adult ICUs in NHSL.

\section{Material and method:}

The audit was carried out from $1^{\text {st }}$ to $31^{\text {st }}$ March 2011, in 5 adult ICUs - Medical (MICU), Surgical (SICU), Cardiac (CICU), Trauma (TICU) and Obstetric (OICU) attached to NHSL.

\section{Ethical consideration:}

Confidentiality was maintained. The care of patients was not affected by the audit.

\section{Audit setting and the population:}

There were altogether 31 beds (SICU-8, MICU-8, OICU-3, TICU-6 and CICU-6) in the 5 ICUs. Admission policy and the type of patients were different from one another as each of these were specialized ICUs. Total study population comprised 90 patients.

\section{Data collection and the method:}

Data were obtained through data collection forms which were filled by a postgraduate trainee in anaesthesiology and critical care, appointed to the relevant ICU during that particular period. Patients' records, drug chart and direct questioning from the conscious patients were the added sources of data.

The risk factors looked for were as follows: Age $>60$ yrs, obesity (BMI>30), history of VTE, acquired or inherited bleeding disorders, history of malignancy and treatment, oral contraceptives or hormone replacement therapies, history of peripheral vascular disease, prolonged immobility, recent major surgery and history of prolonged travel before or after surgery. ${ }^{1}$

\section{Analysis of the data:}

Following areas were addressed during the analysis of the data.

Risk factors for VTE

Contraindications for VTE prophylaxis

Common methods of VTE prophylaxis

\section{Results:}

During the period of one month 90 patients were audited.

Table 1: Distribution among ICUs

\begin{tabular}{|lll|}
\hline Type of ICU & Number & $\%$ \\
MICU & 21 & 24 \\
OICU & 19 & 21 \\
TICU & 19 & 21 \\
CICU & 19 & 21 \\
SICU & 12 & 13 \\
\hline \multicolumn{2}{c}{ Total patients: 90}
\end{tabular}

Table 2

\begin{tabular}{|lll|}
\hline Gender & Number & $\%$ \\
Male & 48 & 53 \\
Female & 42 & 47 \\
\hline \multicolumn{2}{c}{ Total patients: 90} \\
\hline
\end{tabular}

Table 3

\begin{tabular}{|ll|}
\hline Age Group & Number \\
$10-20$ years & 10 \\
$20-40$ years & 36 \\
$40-60$ years & 26 \\
$60-80$ years & 17 \\
$>80$ years & 01 \\
\hline
\end{tabular}

Total patients: 90 
Table 4: Indication for admission

\begin{tabular}{|c|c|c|}
\hline Post operative & $\begin{array}{c}\text { Number } \\
39\end{array}$ & $\begin{array}{l}\% \\
43.0\end{array}$ \\
\hline $\begin{array}{l}\text { Systemic. } \\
\text { dysfunction }\end{array}$ & 20 & 22.0 \\
\hline Road traffic accidents + head injury & 19 & 21.2 \\
\hline Pregnancy + Heart Disease & 8 & 8.8 \\
\hline Post Arrest & 3 & 3.3 \\
\hline $\begin{array}{l}\text { Sepsis following normal vaginal } \\
\text { delivery }\end{array}$ & 1 & 1.1 \\
\hline
\end{tabular}

Total patients: 90

Table 5: Distribution of operative condition

\begin{tabular}{|lc|}
\hline Operative condition & Number \\
Cardiac & 19 \\
Obstetrics \& Gynecology & 17 \\
Abdominal & 09 \\
Trauma and orthopedic & 05 \\
Other general & 03 \\
\hline
\end{tabular}

Table 6: Prevalence of VTE risk factors

\begin{tabular}{|lll|}
\hline Risk factor & Number & $\%$ \\
Critical care admission & 90 & 100 \\
$\begin{array}{l}\text { Expected significant reduction } \\
\text { mobility }\end{array}$ & 56 & 62 \\
Medical co-morbidities & & \\
Age $>60$ years & 50 & 55 \\
Obesity: BMl $>30$ & 16 & 18 \\
Active cancer / cancer treatment & 11 & 12 \\
Dehydration & 04 & 4.4 \\
Varicose veins / thrombophebitis & 01 & 1.1 \\
\hline
\end{tabular}

Table 7: Prevalence of contraindications for pharmacological prophylaxis

\begin{tabular}{|lll|}
\hline Risk factor & Number & $\%$ \\
Derangement of liver function & 19 & 35.8 \\
Platelet $<75000$ & 11 & 20.7 \\
Active bleeding & 07 & 13.2 \\
Acute stroke & 04 & 7.5 \\
Uncontrolled systolic hypertension & 02 & 3.75 \\
Thyroidectomy & 02 & 3.75 \\
\hline
\end{tabular}

Table 8: Prevalence of contra indications for the use of anti - embolic stockings

\begin{tabular}{|lll|}
\hline Risk factor & Number & $\%$ \\
Severe leg oedema & 7 & 7.7 \\
Peripheral arterial bypass graft & 7 & 7.7 \\
Cardiac failure & 6 & 6.6 \\
Local dermatitis / Gangrene & 5 & 5.5 \\
Peripheral vascular disease & 3 & 3.3 \\
Peripheral neuropathy / sensory & 3 & 4.4 \\
impairment & & \\
Venous ulcer / wound & 4 & 4.4 \\
\hline
\end{tabular}

Table 9: Usage of VTE prophylaxis

\begin{tabular}{|lll|}
\hline & Number & $\%$ \\
Nos & 51 & 57 \\
& 39 & 43 \\
\hline
\end{tabular}

Table 10: Type of VTE prophylaxis

\begin{tabular}{|lll|}
\hline & Number & $\%$ \\
Pharmacological only & 17 & 44 \\
Mechanical only & 09 & 23 \\
Total 39 & 13 & 33 \\
\hline
\end{tabular}

Table 11: Prevalence of risk factors

\begin{tabular}{|lll|}
\hline Number of risk factors & Number & $\%$ \\
2 risk factors & 39 & 43 \\
3 risk factors & 24 & 27 \\
4 risk factors & 21 & 23 \\
$>4$ risk factors & 6 & 7 \\
\hline
\end{tabular}

Table 12: Risk factor prevalence in group who received VTE prophylaxis

\begin{tabular}{|lll|}
\hline Number of risk factors & Number & $\%$ \\
2 risk factors & 17 & 44 \\
3 risk factors & 08 & 23 \\
4 risk factors & 12 & 33 \\
$>4$ risk factors & 02 & 5 \\
\hline
\end{tabular}

Total patients: 39

Table 13: Risk factor prevalence in group who did not receive VTE prophylaxis

\begin{tabular}{|lll|}
\hline Number of risk factors & Number & $\%$ \\
2 risk factors & 25 & 49 \\
3 risk factors & 13 & 25 \\
4 risk factors & 09 & 18 \\
$>4$ risk factors & 04 & 08 \\
\hline
\end{tabular}

Total patients: 51

Table 14: Contraindications for VTE prophylaxis in patients who were not on VTE prophylaxis

\begin{tabular}{|c|c|c|}
\hline & Number & $\%$ \\
\hline $\begin{array}{l}\mathrm{C} / \mathrm{f} \text { for pharmacological } \\
\text { prophylaxis }\end{array}$ & 11 & 21 \\
\hline $\mathrm{C} / \mathrm{l}$ for mechanical prophylaxis & 04 & 08 \\
\hline $\mathrm{C} / \mathrm{l}$ for both & 04 & 08 \\
\hline C/l for None & 32 & 63 \\
\hline
\end{tabular}

Total patients: 51 
Table 15: Pharmacological prophylaxis

\begin{tabular}{|lc|}
\hline Anticoagulant Agent & No. \\
Enoxaparin & \\
$15 \mathrm{mg}$ daily & 1 \\
$20 \mathrm{mg}$ daily & 4 \\
$30 \mathrm{mg}$ daily & 1 \\
$40 \mathrm{mg}$ daily & 17 \\
$60 \mathrm{mg}$ bd & 1 \\
$60 \mathrm{mg}$ daily & 1 \\
& \\
Tinzaparine & \\
$3500 \mathrm{U}$ daily & 3 \\
$7000 \mathrm{U}$ daily & 1 \\
Heparin $5000 \mathrm{U}$ bd & 1 \\
\hline
\end{tabular}

Table 16: Time to commencement of pharmacological prophylaxis post operatively

\begin{tabular}{|lc|}
\hline Time frame-post op & Number of patients \\
$4-8 \mathrm{H}$ & 2 \\
$8-12 \mathrm{H}$ & 7 \\
$12-24 \mathrm{H}$ & 3 \\
$>24 \mathrm{H}$ & 4 \\
\hline
\end{tabular}

Total patients: 16

Discussion: Out of total 90 patients, least number was recorded from the SICU 13\% (12 patients). This might be due to the lower rate of patient turnover in SICU during that time. 20-40 year age group contributed mainly for the study population (40\%). This may be due to the contribution from the child bearing age group from the OICU and its higher patient turnover. Majority (49\%) of the study population was admitted following surgery. This is probably as we have audited 4 surgical based ICUs. Among other common reasons for the admission to the critical care unit were, systemic illness with organ failure (22\%), trauma (15.5\%) and heart disease complicating pregnancy $(8.8 \%)$.

Among the post operative population, following surgeries belonged to the high risk category for DVT risk; major general surgeries (oesophagectomy, abdomino perineal resection, thyroidectomy and Whipple's procedure), orthopedic (kyposcoliosis correction) and major vascular surgeries (kidney transplant) ${ }^{1}$. Except 2 thyroidectomies and 1 kidney transplant all other patients had received both mechanical and pharmacological prophylaxis. Two patients who underwent thyroidectomy had not received any mode of VTE prophylaxis (they had been mobilized out of bed on post operative day 1) while the kidney transplant patient had received only pharmacological prophylaxis.

According to the 2006 October NICE guidelines on VTE prophylaxis for high risk surgical patients, all patients who are undergoing high risk surgeries (major general surgery, vascular surgery, gynecological surgery, urological surgery, neurological surgery and cardiac surgery) should receive mechanical prophylaxis. If these patients carry one or more risk factors they should be offered both mechanical and pharmacological prophylaxis (low molecular weight heparin / fondaparinux). Patients with elective major orthopedic surgeries should receive both type of prophylaxis irrespective of the number of the risk factors ${ }^{1}$.

Cardiac surgeries fall in to the high risk for VTE, but majority of those patients were not put on VTE prophylaxis. Early mobilization and the fact that patients with valvular surgeries were started on anticoagulants during immediate post operative period, might be the reasons for why they did not receive VTE prophylaxis.

Majority of patients admitted to a critical care unit has a major risk factor for VTE, and most have multiple risk factors. Many of these thrombosis risk factors precede the ICU admission, while others develop during the course of ICU stay. The importance of each of these clinical risk factors is unknown, as is the role of inherited or acquired coagulation system abnormalities. However, adequately powered studies using multiple logistic regression analysis to determine the independent predictors for thrombosis in critically ill patients have not yet been conducted. ${ }^{3}$ A double-blind, placebo-controlled, randomized trial on DVT prophylaxis has been performed in 791 medical ICU patients; thrombosis was detected by Doppler ultrasound examinations performed every 3 days. DVT was diagnosed in $31 \%$ of the 390 control patients, and $5 \%$ had PE. ${ }^{3}$

In our study population (90 patients) $57 \%$ (51 patients) had not received VTE prophylaxis while $43 \%$ (38 patients) had received it. Looking for the distribution of the risk factors in the study population 39 patients (43\%) had at least 2 risk factors for VTE. 6 patients ( $8 \%$ ) were found to 
have $>4$ risk factors for VTE. But only 3 out of those 6 patients received VTE prophylaxis. 17 out of 39 patients who received VTE prophylaxis had 2 risk factors for VTE while 25 patients who did not receive VTE prophylaxis, also had 2 risk factors for VTE.

Despite the paucity of critical care-specific data about thromboembolism, the risks of VTE in other patient groups, including surgical, trauma/spinal cord injury, and medical patients, are well established and are relevant to those in critical care units. Objectively confirmed DVT rates were found to be in the range of 10 to $80 \%$ for patients admitted to ICUs or following trauma, neurosurgery, or spinal cord injury in a recent systematic review. ${ }^{3}$

Acute liver failure (21\%) was the commonest risk factor for bleeding (contra indication for pharmacological VTE prophylaxis). thrombocytopenia and INR $>2$ were the other risk factors encountered. Above 3 contra indications were consistently found in the patients with multi organ dysfunction following sepsis highlighting the importance of frequent assessment of the risk and benefit of pharmacological VTE prophylaxis among the critical care population.

Peripheral arterial bypass graft and severe leg oedema were the two identified commonest contra indications for the use of anti embolic stockings.

One of the objectives of this study was to assess the incidences of VTE prophylaxis usage in the critical care set up in NHSL Colombo. 57\% (51 patients) had not been given any form of VTE prophylaxis. Out of these 51 patients $62.7 \%$ (32 patients), had no contraindications for pharmacological or mechanical VTE prophylaxis methods. $22 \%$ of patients had contra indications for pharmacological prophylaxis, $8 \%$ had contra indications for mechanical prophylaxis and another $8 \%$ had contra indications for both.

$68.7 \%$ of patients who had not been given VTE prophylaxis had more than one risk factor for VTE (5 patients had $>4$ risk factors for VTE), while the other 10 patients had only one (admission to critical care unit).

- 7 out of these 10 patients were mobilized out of bed within 24 hours.

- 6 of them belonged to $20-40 \mathrm{yr}$ age group.

- None of them had a BMI $>30 \mathrm{~kg} / \mathrm{m}^{2}$

- 2 were post thyroidectomy patients

- 01 patient was with a combined spinal epidural

- 02 were for observation following trauma

These might be the reasons why they were not given VTE prophylaxis. 39 patients out of this 51 (76.4\%) were audited during the $1^{\text {st }}$ and $2^{\text {nd }}$ day of admission to the critical care unit. This might be another reason why these patients were not started on VTE prophylaxis.

In 39 patients who were treated with VTE prophylaxis, $43.5 \%$ (17 patients) received only pharmacological prophylaxis as the mechanical devices were not available. Unavailability of the mechanical VTE prophylaxis devices (graduated compression stockings, sequential compression devices, foot pumps) in critical care units is one of the draw backs which we think need to be addressed.

Enoxaparin 40mg daily was the regime used mostly irrespective of the patient's body weight. Only 2 patients were treated with calculated dose according to the BW. Enoxaparin 60mg daily and $60 \mathrm{mg}$ twice a day were the other common regimes used. Currently accepted anticoagulant agents in VTE prophylaxis are unfractionated heparin, low molecular weight heparin, oral direct factor Xa inhibitors, warfarin, aspirin and fondaparinux. ${ }^{1,2}$

Graduated compression stockings are also considered as effective as pharmacological prophylaxis. Other forms of mechanical methods include electrical stimulation of the calf muscles and intermittent external pneumatic compression devices. ${ }^{1,2}$

Most of the post operative patients who were started on VTE prophylaxis were started 8-12 hours after surgery $(43.7 \%)$ which correctly matches with the guidelines. Starting VTE prophylaxis in $12-24 \mathrm{hr}$ post op period was $18.7 \%$ and $>24 \mathrm{hr}$ post op period $25 \%$. One of the reasons for delayed start of pharmacological prophylaxis 
was waiting for the laboratory results of the coagulation screen.

\section{Conclusion:}

We understand that there are possible challenges and barriers in providing ideal VTE prophylaxis in ICUs in Sri Lanka and it is important to consider the risks - benefit in critically ill patients with multiple risk factors for other fatal conditions.

We suggest:

1. Team work, discussions, workshops, seminars and such awareness programs for all categories of ICU staff towards updating the knowledge base and reaching a consensus decision towards a standardized printed guideline for VTE prophylaxis for critically ill patients, if necessary with modifications to suit each separate specialized ICU category.

2. An essential component of the assessment of all ICU admissions should be a review ofthromboembolic risks and a consideration of thromboprophylaxis.

3. Decisions regarding the initiation of prophylaxis and selection of the specific method of prophylaxis should be individualized and based on each patient's risks for bleeding and thrombosis.

Prophylaxis should be reviewed daily and changed, if necessary, taking into consideration each patient's overall clinical status on that particular day.
Adherence to the thromboprophylaxis policy should be assessed using audits and, improvement efforts should be undertaken.

Acknowledgement: I would like to thank Dr (Mrs) Nalini Rodrigo, Senior Consultant Anaesthetist, for her advice and support during the review process of the paper and Dr Buddhika Habaragamuwa, Consultant Anaesthetist, Base hospital, Mawanella for provision of the questionnaire and Dr. T S Jayaweera, Dr L A W M D Atapattu, Dr S L Ranasinghe (post graduate trainees in anaesthesiology) for their support with data collection.

\section{References}

1. Prevention on Venous Thromboembolism in patients undergoing high risk surgical procedures: NICE guide lines: October 2006

2. Prevention and management of Venous Thrombo Embolism: SIGN guide lines: December 2010

3. Greets W.H, Rita S, Prevention of Venous Thrombo Embolism in ICU: Chest 2003; 124:357363

4. Attia J, Ray J G, Cook D J, Douketis J, Ginsberg J S, Greets W H. Deep Vein Thrombosis and Its Prevention in Critically Ill Adults. Arch Intern Med.2001:161:1268-1279

5. Limpusa A, Chaboyer W, McDonald E, Thalib L Mechanical Thromboprophylaxis in critically ill patients: American Journal of Critical care: 2006;15:402-412 